\title{
Rapid Electrochemical Analysis of Plant and Synthetic Indigo Dyes
}

\author{
Qiong Wang ${ }^{1,2}$, Boyuan $\mathrm{Fan}^{3}$, Dongling $\mathrm{Li}^{1,2}$, Zenglai Xu ${ }^{1,2, *}, \mathrm{Li} \mathrm{Fu}^{3, *}$ Aiwu Wang ${ }^{4}$ \\ Yuhong Zheng ${ }^{1,2}$, Jiangwei $\mathrm{Zhu}^{5}$ \\ ${ }^{1}$ Institute of Botany, Jiangsu Province \& Chinese Academy of Sciences (Nanjing Botanical Garden \\ Mem. Sun Yat-Sen), Nanjing 210014, P.R. China \\ ${ }^{2}$ The Jiangsu Provincial Platform for Conservation and Utilization of Agricultural Germplasm, \\ Nanjing 210014, PR China \\ ${ }^{3}$ College of Materials and Environmental Engineering, Hangzhou Dianzi University, Hangzhou, \\ 310018, P.R. China. \\ ${ }^{4}$ Center for Advanced Material Diagnostic Technology, Shenzhen Technology University, Shenzhen \\ 518118, P.R. China \\ ${ }^{5}$ Co-Innovation Center for Sustainable Forestry in Southern China, Nanjing Forestry University, \\ Nanjing 210037, P.R. China \\ *E-mail: 13555655293@qq.com; fuli@hdu.edu.cn
}

doi: $10.20964 / 2021.04 .56$

Received: 12 December 2020 / Accepted: 3 February 2021 / Published: 28 February 2021

\begin{abstract}
Plant indigo was a very important dye historically, but it was later replaced by synthetic indigo. In recent years, plant indigo has been recognized for its artistic value and reduced environmental impact. However, the price of plant indigo is much higher than that of synthetic indigo. In this work, we propose an electrochemical method for quickly distinguishing plant indigo from synthetic indigo. This method uses an electrochemical fingerprint technique. Using the different fingerprints of plant indigo and synthetic indigo, corresponding indexes were developed for identification. In addition, this technology can also distinguish plant indigo and synthetic indigo mixed in different ratios. This rapid technology requires no large-scale instruments and has great potential for dye analysis.
\end{abstract}

Keywords: Electroanalysis; Indigo dyes; Fast identification; Fingerprints; Differential pulse voltammetry

\section{FULL TEXT}

(C) 2021 The Authors. Published by ESG (www.electrochemsci.org). This article is an open access article distributed under the terms and conditions of the Creative Commons Attribution license (http://creativecommons.org/licenses/by/4.0/). 